\title{
APLICACIÓN DEL MÉTODO KANBAN A UN SOFTWARE CRM BASADO EN Plataforma Open SOURCE Vtiger
}

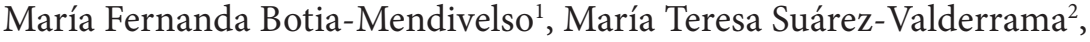 \\ Oswaldo Alberto Romero-Villalobos ${ }^{3}$ \\ ${ }^{1}$ Ingeniera Industrial. Asistente de investigación. Correo electrónico: mbotiam@udistrital.edu.co \\ ${ }^{2}$ Ingeniera Industrial. Asistente de investigación \\ ${ }^{3}$ Magister en Ingeniería Industrial. Docente de tiempo completo e investigador \\ Grupo de Investigación en Interoperabilidad de Redes Académicas (GIIRA), Facultad de Ingeniería, \\ Universidad Distrital Francisco José de Caldas, Bogotá, Colombia
}

Recibido: 10 de enero del 2016 Aprobado: 10 de julio del 2016

Cómo citar este artículo: M. F. Botía-Mendivelso, M. T. Suárez-Valderrama, 0. A. Romero-Villalobos, "Aplicación del método Kanban a un software crm basado em plataforma Open Source Vtiger, Ingeniería Solidaria, vol. 12, no. 20, pp. 9-22, oct. 2016. doi: http://dx.doi.org/10.16925/in.v19i20.1412

Resumen. Introducción: este artículo es producto de la investigación "Importancia de la simplicidad Software CRM desde la perspectiva del usuario", dirigida por el Grupo de Investigación en Interoperabilidad de Redes Académicas (GIIRA) de la Universidad Distrital Francisco José de Caldas realizada durante el 2015. Metodología: se implementó el método Kanban en la plataforma basada en Open Source Vtiger y se hizo seguimiento a 20 empresas usuarias, a través de recolección de datos sobre estadísticas de uso para compararlas con la información previa obtenida durante el primer semestre del 2015, periodo en el cual la plataforma presentaba una interfaz similar a la de Vtiger. Resultados: el $85 \%$ de las empresas aumentó significativamente el uso de la plataforma hasta en un $310 \%$; el $10 \%$ no tuvo variaciones significativas y el $5 \%$ restante registró un menor uso. Conclusiones: la implementación del método Kanban en el software Customer Relationship Management (CRM) permite tener acceso a la información de gestión comercial de manera organizada, resaltando en primer plano datos de interés fácilmente concluyentes, al igual que redireccionar la información complementaria si es necesario.

Palabras clave: cliente, gestión, Kanban, método, software. 


\title{
IMPLEMENTATION OF THE KANBAN METHOD TO A CRM Software based in the Open Source Platform Vtiger
}

\begin{abstract}
Introduction: This article is the result of the research "Importance of the CRM Software simplicity from the user's perspective", conducted by the Research Group in Interoperability in Academic Networks (GIIRA) of the District University Francisco Jose de Caldas, during the year 2015. Methodology: The Kanban method was implemented in the Open Source Vtiger-based platform, and 20 user companies were monitored for data collection in order to compare their usage with the information obtained during the first semester of 2015. During that period, the platform had an interface similar to Vtiger's. Results: $85 \%$ of the companies increased their use of the platform significantly up to $310 \%$. The other $10 \%$ did not present significant variations, and the remaining $5 \%$ registered a decrease in their use of the platform. Discussion: The implementation of the Kanban method in the Customer Relationship Management (CRM) software allows access to commercial management information in an organized manner, thus highlighting key facts that are easily conclusive, and redirecting additional information, if necessary.
\end{abstract}

Keywords: customer, management, Kanban, method, software.

\section{APLiCaÇão do MÉtodo Kanban A UM SOFTWARE CRM baseado na plataforma Open Source Vtiger}

Resumo. Introdução: este artigo é produto da investigação "Importância da simplicidade Software CRM desde a perspectiva do usuário", feita pelo Grupo de Investigação em Interoperabilidade de Redes Académicas (GIIRA), da Universidade Distrital Francisco José de Caldas, durante o ano 2015. Metodologia: foi aplicado o método Kanban na plataforma baseada em Open Source Vtiger fazendo acompanhamento a 20 empresas usuárias, através de coleta de dados sobre estatísticas de uso para serem comparadas com a informação previa obtida durante o primeiro semestre de 2015, durante o qual a plataforma apresentava uma interface semelhante à do Vtiger. Resultados: determinou-se que $85 \%$ das companhias aumentou significativamente o uso da plataforma até $310 \%$; $10 \%$ não teve variações significativas e $5 \%$ restante registrou um uso menor. Conclusões: a implementação do método Kanban no software Customer Relationship Management (CRM) permite ter acesso à informação de gestão comercial de maneira organizada, destacando em primeiro plano dados de interesse facilmente concludentes, igual do que redirecionar a informação complementar se for preciso.

Palavras chave: cliente, gestão, Kanban, método, software. 


\section{Introducción}

En la actualidad, el cliente no se limita a la oferta de una empresa determinada. Ahora tiene a su disposición herramientas que le permiten indagar, con un solo clic, las diferencias entre las ofertas disponibles, siendo necesario para las organizaciones emplear mecanismos que reduzcan la brecha entre empresa y cliente; por tanto, las empresas buscan soluciones tecnológicas que permitan mantener $\mathrm{y}$ aumentar la eficiencia de su negocio, para garantizar así información en tiempo real sobre preferencias, comportamientos y requerimientos de los consumidores.

Los sistemas de información soportados en cloud computing (en español, computación en la nube) representan soluciones inmediatas a dichas necesidades empresariales. Para el desarrollo de este artículo, nos centraremos en software como servicio (Software-as-a-Service [SAAS]), el cual es un nuevo modelo de entrega de software basado en ofrecer aplicaciones complejas desde Internet [1]. Los sistemas Customer Relationship Management (CRM) soportados en la web son en la actualidad uno de los SAAS más implementados. Los objetivos de CRM Como SAAS son optimizar los procesos de marketing y reducir costos de operación en ventas, mejorar relaciones empresa-cliente, brindar soporte para toma de decisiones, aumentar rentabilidad, entre otros; desplazando aquí la administración de base de datos de los clientes basadas en hojas de cálculo y el flujo de información limitada al uso de correo electrónico.

Para poner en funcionamiento un software de servicio es indispensable la participación de dos actores: proveedor y usuario. El primero se encarga de crear la aplicación en la red, alojar la información en servidores y garantizar su disponibilidad, realizar actualizaciones y mantenimientos pertinentes. En el caso de los usuarios, SAAS está diseñado para ser multitenant ${ }^{1}$, es decir, en una sola instancia de tiempo de ejecución de la aplicación varias organizaciones y usuarios pueden hacer uso de ella [2]. Uno de los desafíos para los desarrolladores de SAAS es crear ambientes de trabajo amigables para el usuario, que reduzcan procesos y que su aprendizaje sea particularmente intuitivo.

Este artículo se realizó con base en información recolectada en la empresa Data CRM,

1 Se refiere a múltiples usuarios. que adapta el CRM Vtiger a las condiciones de las empresas colombianas, en cuanto a funcionalidad, beneficios y costos [3]. El objetivo de este artículo es evaluar, desde la perspectiva del usuario, la simplicidad en el uso de un sistema CRM mediante la adopción del método Kanban, definido como un mecanismo de control de flujo de material, que controla la cantidad y el momento adecuado de la producción necesaria [4]. El método de Kanban se adapta al software Data CRM a partir del código libre de la plataforma Vtiger, y permite así al usuario visualizar en la pantalla el estado del proceso de marketing y acceder fácil y rápido a la información requerida. A partir de la propuesta para mejorar el desarrollo de la aplicación, se estiman medidas de desempeño por medio de estadísticas de uso.

El artículo se complementa de la siguiente manera: en las primeras secciones se contextualizan los conceptos de CRM, software como servicio y CRM ofertado como software de servicio. Las siguientes secciones se enfocan en el análisis práctico del desarrollo del artículo: aplicación del método Kanban al software CRM, presentación y análisis de estadísticas de uso y conclusiones.

\section{Metodología}

\subsection{Customer relationship management}

En el mundo empresarial se están usando nuevas prácticas de negocios al cambiar la perspectiva hacia los factores externos; un ejemplo de esto es la aplicación de CRM, una herramienta de gestión que busca que la organización tenga una relación efectiva con los clientes y construir relaciones a largo plazo. Esto para llevar a la organización a realizar procesos orientados al cliente, que le permitan tener la capacidad de atender, detectar sus necesidades y lograr su satisfacción.

Las empresas deben tener la capacidad de ofrecer a los consumidores lo que demanden. De acuerdo con los principios de CRM, la organización debe interactuar con el cliente, haciendo seguimiento continuo de la experiencia con los productos, documentando y analizando la información obtenida con el objetivo de encontrar patrones de comportamiento y usar este conocimiento para aumentar las ventas. Como lo mencionan Puente y Cervilla [5], el CRM provee oportunidades resaltantes para usar 
datos e información para entender a los clientes y crear valor con ellos. Asimismo, Robert et al. [6] sostienen que la comunicación en CRM y el aporte del cliente no se limita a la recepción de información sobre el mismo, aunque este ya es un componente que a la mayoría de las organizaciones les puede resultar innovador; sin embargo, el ideal es tener una retroalimentación constante.

Pérez [7] afirma que CRM se articula como un concepto surgido a la luz de la evolución tecnológica, consistente en un rediseño del negocio y sus procesos en torno a las necesidades y deseos del cliente. El CRM une el potencial de las estrategias de marketing relacional con la tecnología de información (TI) para crear relaciones rentables de largo plazo con los clientes y otras audiencias clave [8]. $\mathrm{Al}$ adoptar CRM las empresas adecúan su estrategia a un enfoque centrado al cliente en todos los ámbitos, tanto funcional como técnico, utilizando herramientas tecnológicas y preparando al personal para tener una visión 360 grados del cliente [9]. Es decir, CRM no es exclusivo del área comercial de una compañía, sino que debe convertirse en una estrategia aplicada en toda la organización.

Las ventajas que tiene implementar CRM $[10$, 11] son:

- Mejorar la tasa de satisfacción de los clientes.

- La creación de valor para los clientes.

- El aumento de la lealtad de los clientes para las empresas.

- Mejorar el servicio al cliente.

- Cerrar las ventas más rápido.

- Simplificar los procesos de marketing y ventas.

- Descubrir nuevos clientes y aumentar los ingresos de clientes.

Según Buttle [12], existen cuatro tipos de CRM: operativo, analítico, colaboración y estratégico, los cuales se describen a continuación:

- CRM operacional: automatiza y mejora los procesos del negocio proporcionando soporte al cliente y buscando trabajar de cara al público. Además, implica el uso de la tecnología en las funciones del negocio-ventas, marketing y servicio [13].

- CRM analítico: hace tangible los beneficios de una política CRM, como por ejemplo identificar a los clientes más rentables, aquellos con una mayor tendencia a comprar o con un mayor potencial [14]. En este se hace el análisis de toda la información recogida en el CRM operacional.

- CRM colaborativo: incluye soluciones que aumentan la capacidad del cliente para colaborar en procesos empresariales (sistemas autoservicio) [15]; por lo tanto, la empresa busca tener herramientas que le permitan al cliente interactuar (por ejemplo los canales de Internet de un banco).

- CRM estratégico: define los procesos de relación con clientes a través de los distintos estados: captación, prospección, compra y renovación, hasta convertirlo en prescriptor de la marca [16].

También cabe mencionar una estrategia que está tomando mucha fuerza y que puede catalogarse como otro tipo de CRM, el CRM social que integra el CRM y el poder de las comunidades en línea [17]. El valor de las redes sociales en las actividades de CRM reside en la gran cantidad de personas que utilizan los medios sociales para comunicarse y la amplia información que es generada en ellas [18].

\subsubsection{Software como servicio}

La invención y comercialización de Internet no solo implicó el desarrollo competitivo y servicios de redes privadas, sino también el desarrollo de productos comerciales aplicando la tecnología [19] de la red informática de comunicación. La computación en la nube representa uno de los avances tecnológicos más actuales y relevantes derivados del desarrollo web. Según el National Institute of Standards and Technology (NIST), el cloud computing es un modelo para permitir acceso ubicuo a la red bajo demanda a un conjunto compartido de recursos informáticos configurables (redes, almacenamiento, aplicaciones y servicios) que pueden ser rápidamente aprovisionados y liberados con un mínimo esfuerzo de gestión o interacción con un proveedor de servicios. En cloud computing se distinguen tres categorías fundamentales se servicio [20]:

- Software como servicio (SaAS).

- Plataforma como servicio (PaAS).

- Infraestructura como servicio (IAAS).

Sin embargo, entidades importantes en la implementación de servicios en la nube como Amazon, 
Google, Salesforce.com y Microsoft añaden nuevas categorías [21]:

- Base de datos como servicio (DAAS)

- Business Process as a Service (BPAAS)

SAAS es un modelo de distribución de software, en el cual las aplicaciones de software están alojadas por un proveedor de servicio y puestos a disposición de los clientes a través de Internet [22]. El usuario final no requiere ningún tipo de infraestructura propia para la implementación y ejecución de la plataforma, tampoco incurre en procesos de instalación ni mantenimiento; sin embargo, sí pagan tarifas de uso, con la facilidad de acceder de forma remota a las aplicaciones y bases de datos que residen en servidores de propiedad de los proveedores [23]. Estos pagos generalmente se realizan de forma mensual y el monto se relaciona con el número de usuarios que ejecute el programa instalado en la nube.

Un sistema de aplicación SAAS bien diseñado tiene tres propiedades principales: orientada al cliente, elasticidad y eficiencia multiusuario [24], y se fundamenta en ciertas características generales, como el acceso vía web, el soporte y la gestión de actualizaciones por parte del proveedor, el precio de suscripción y los requisitos particulares limitados [25]. De esta manera, transforma la tecnología convencional y logra ser un método exitoso para el desarrollo de los procesos dentro de las empresas gracias a las ventajas que ofrece, mencionadas a continuación $[2,26,28]$ :

- Tiempo corto de implementación en la empresa.

- Manejo de economía a escala. Los usuarios comparten los recursos de infraestructura como ordenador, servidores y dispositivos de almacenamiento de datos.

- Los clientes no tienen que gastar tiempo y dinero en mejoras y despliegue de software.

- La información se encuentra consolidada en un solo sitio (en la nube), disponible en tiempo real y con acceso desde cualquier parte del mundo.

Actualmente, SAAS es el más importante y prometedor modelo de servicio en la nube y las previsiones recientes indican la continuación de esta tendencia para los próximos años [29]. Según Forrester, citado en [30], el mercado computacional de la nube fue valorado en $\$ 40,7$ mil millones en el 2010 y se espera que crezca a $\$ 241$ mil millones en el 2020.

\subsection{Customer Relationship Management ofertado como un servicio}

CRM software es una aplicación ejecutada desde la nube que hace referencia al análisis de ventas, marketing y servicio al cliente [31]. Según Petkovic [32], el CRM no es una tecnología de la información, pero sí la requiere, por lo tanto, las empresas deben buscar implementar sistemas de información que les permitan organizar y orientar su negocio hacia el cliente. Una motivación principal para que una empresa implemente aplicaciones de CRM es comprender mejor las necesidades de los clientes. Mediante la organización y el uso de esta información, las empresas pueden diseñar y desarrollar mejores productos y servicios [33].

En Colombia aumenta el uso de aplicaciones para el manejo y control de los negocios, siendo las más relevantes las orientadas a sistemas de planificación de producción y a administrar la relación con el cliente (CRM) [34]. Según Eurostat, en el 2014 el $20 \%$ de las empresas de la unión europea (EU) han utilizado software para analizar información sobre clientes con fines de marketing [35].

Según la revista Forbes, en el 2013, el $41 \%$ de todos los sistemas de CRM estaba basado en SAAS, y ascendió al $47 \%$ en el $2015[36,37]$. En la figura 1 se ilustran los principales proveedores de software de servicio y su participación en el mercado global.

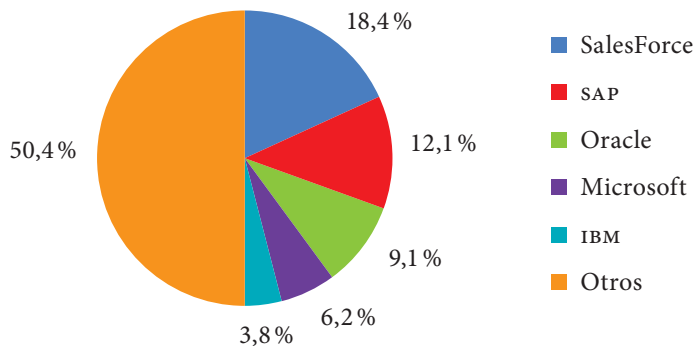

Figura 1. Participación del SAAS más usados en la economía global en el 2014

Fuente: [36]

\subsubsection{Salesforce}

Salesforce es el software de servicios líder en el mercado, reconocido por permitir la movilización 
y aumento de ingresos de la empresa. Permite acceder de forma fácil y rápida al historial y registro de interacciones, preferencias actuales, detalles de la infraestructura, seguimiento en redes sociales, interacciones en ventas (todo enfocado al cliente).

Según un informe de la compañía, en el 2013, los clientes reportaron $36 \%$ de aumento en la productividad de ventas; $25 \%$, en los procesos de ventas; $26 \%$, en las tasas de ventas consolidadas; $30 \%$ en los ingresos, y $45 \%$ en la precisión de pronósticos [38].

\subsubsection{SAP}

Para SAP, la innovación en la cadena de suministro está determinada por la inversión en tecnología. SAP CRM alinea todas las actividades y recursos de marketing alrededor de objetivos estratégicos, permite organizar y distribuir grandes volúmenes de datos a altas velocidades con un sistema de interfaz drag-and-drop que resalta datos clave de cada lead, ${ }^{2}$ permite la organización de campañas de marketing multicanal, sistemas analíticos integrados con herramientas de análisis predictivo para anticiparse al comportamiento de clientes a través de reportes y cuadros de mando preconfigurados [39].

\subsubsection{Microsoft}

Microsoft CRM describe sus ventajas competitivas de la siguiente manera: adaptabilidad, basada en tecnología estándar y fiable de la industria; un centro de funciones que permiten acceso a la información con o sin conexión; integración con ofimática; interfaz de usuario que permite integrar las aplicaciones de productividad de Microsoft Office System; ámbito internacional, soporte en varios idiomas y múltiples zonas horarias [40].

\section{$2.2 .4 \mathrm{IBM}$}

Hace tratamiento a CRM como una "jornada", no como un destino. IBM Cuenta con alianzas estratégicas para desarrollar y entregar soluciones que atiendan las necesidades exclusivas de negocios de nuestros clientes. Ofrece, además, servicios aplicativos personalizados, BM Application Management Services para $e$-business y aplicativos tradicionales. Proporciona ofertas para la administración,

2 Persona o compañía que ha demostrado interés en la oferta de la marca. manutención y soporte corrientes de todos sus aplicativos desarrollados de forma personalizada [41].

\subsubsection{Oracle}

Oracle CRM ofrece diferentes soluciones sectoriales especializadas, así como las funciones de mayor alcance posible para que las organizaciones puedan impulsar distintas facetas de su negocio, como las ventas, el marketing, la fidelidad y el servicio; adicionalmente, brinda a las empresas una gama de otros servicios y soluciones que puede disminuir costos, reducir el riesgo, acelerar el valor e incrementar la escalabilidad. Sus características fundamentales son el foco del sector, la innovación, integración de procesos y variedad en opciones de infraestructura [42].

\section{3. ¿Cómo aplicar el método Kanban a un software CRM basado en la plataforma Vtiger?}

Kanban nace en Toyota en los años cincuenta, con el propósito de eliminar elementos innecesarios en su producción, y es utilizado como medio para obtener materiales entre sus procesos y como una forma de aumentar la calidad de sus productos [43]. Es un tablero que pone a la vista la información necesaria para controlar la evolución del producto e involucrar de forma natural a todos los integrantes en la resolución y la gestión de los problemas [44]. Se divide en un lienzo en columnas y unas etiquetas Kanban que contienen información de qué se va a producir, medios que se utilizarán en la producción, cantidad y encargado del proceso [45] (ver figura 2). Una limitante del Kanban tradicional es que se hace en papel y sus datos no se pueden transmitir directamente a un sistema de información [46].

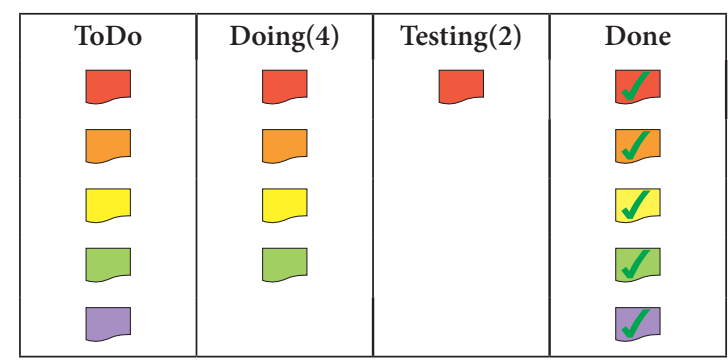

Figura 2. Tablero Kanban

Fuente: [47] 


\subsection{Comparación de la interfaz de usuario de Data CRM con el tablero y sin el tablero Kanban}

Data CRM es una aplicación de Internet que permite organizar el proceso comercial, servicio al cliente, marketing y proyectos, recopilando en un solo lugar toda la información relacionada con los clientes, ya sean contactos, cuentas, cotizaciones o facturas [48]. Además, permite hacer seguimiento a cada fase del desarrollo de un negocio, desde que se envía la cotización hasta la posventa, y así se controlan las actividades que se realizan (las fases de negocio las crea cada empresa según sus necesidades y su estructura).

A continuación se hará una comparación de Data CRM antes y después de la aplicación del método Kanban. Es fundamental dejar claros los siguientes términos utilizados en la aplicación:

- Actividad o evento: se refiere a todas las acciones necesarias para llevar a cabo un negocio (ejemplo: llamar a un cliente, enviar una cotización).
- Contacto: hace referencia a la persona de contacto directo de la empresa con la que se está haciendo la gestión comercial.

- Cuenta o cliente: son empresas o personas con las cuales las compañías usuarias del CRM tienen una relación comercial.

- Oportunidad o negocio: es el planteamiento de un prospecto o de actividad comercial.

Sin la aplicación del método Kanban, en la plataforma CRM los procesos como la creación de un negocio, contacto, actividad o cambiar de fase un negocio pueden ser tediosos, debido a que se deben diligenciar varios formularios (ver figura 3).

Si el usuario requiere ver las oportunidades (negocio), la fase en la que está un negocio o las actividades por realizar, debe ingresar a "negocios", como se evidencia en la figura 4, y dar clic sobre el negocio para obtener información acerca de las actividades. Para cambiar una oportunidad (negocio) de fase debe ir a "cuentas" y modificar el formulario de la figura 3.

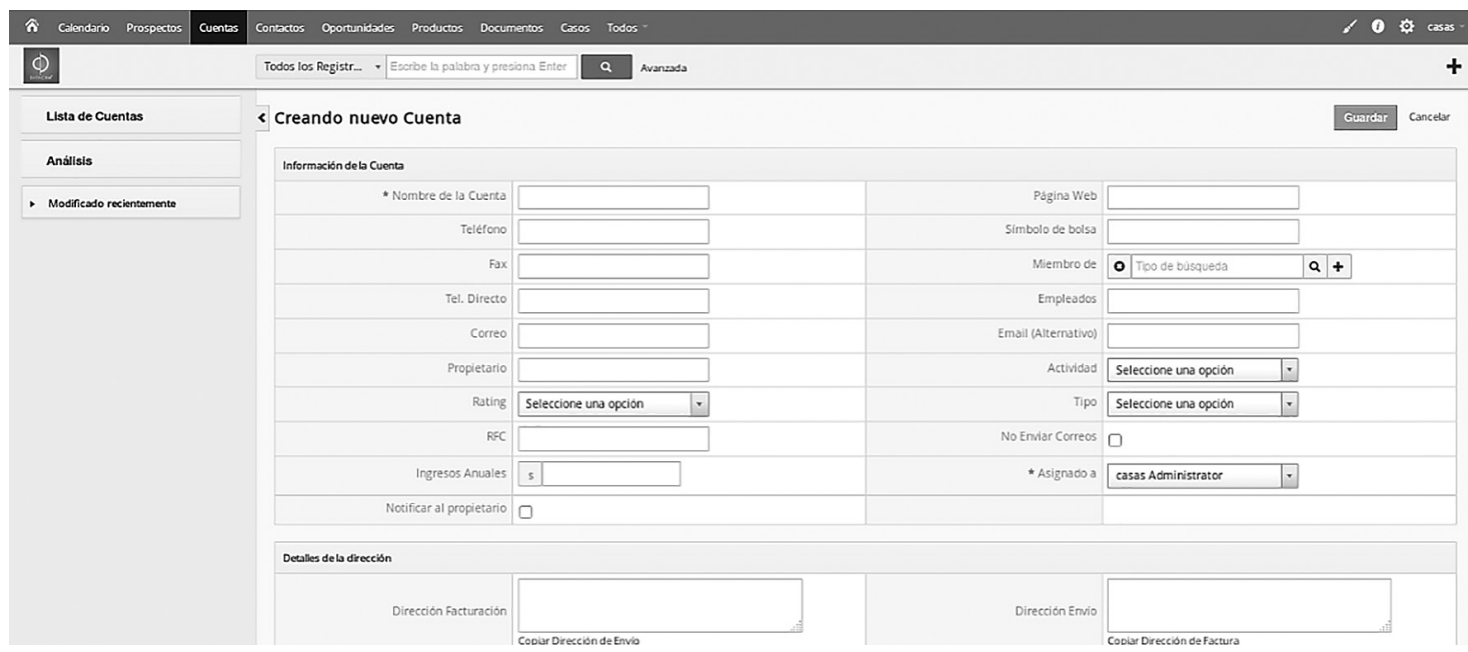

Figura 3. Formulario para crear una cuenta o cliente

Fuente: [49]

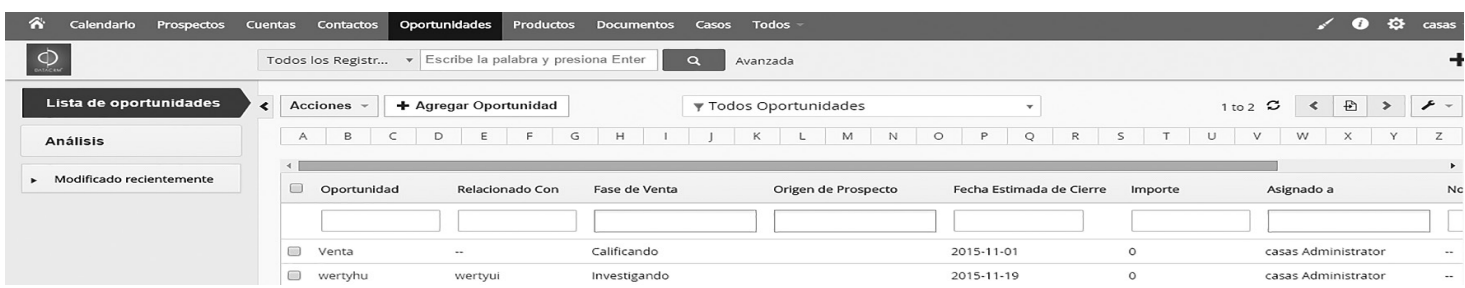

Figura 4. Panel de control de oportunidades (negocios)

Fuente: [49] 
Con la aplicación del método Kanban en un mismo tablero llamado Canvas, se pueden visualizar todos los negocios ordenados por las diferentes fases de negocio, como se indica en la figura 5.

Cada una de las tarjetas contiene información concerniente al nombre del negocio, responsable, valor del negocio, nombre de la empresa, cliente y un indicador que permite diferenciar por medio de colores si la actividad está planeada (verde), está planeada para hoy (azul), está retrasada (amarillo), o el negocio no tiene actividades (rojo), como se evidencia en la figura 6. En el CRM, sin la aplicación de Kanban, no existe ninguna alerta de las actividades planeadas o retrasadas, por lo que es necesario que el usuario revise reiteradamente el calendario.

Para crear un cliente (cuenta), un contacto o un negocio (oportunidad), se puede hacer de una forma más sencilla, entrando a la opción "crear en negocio", y solo es necesario llenar el formulario que se indica en la figura 7.

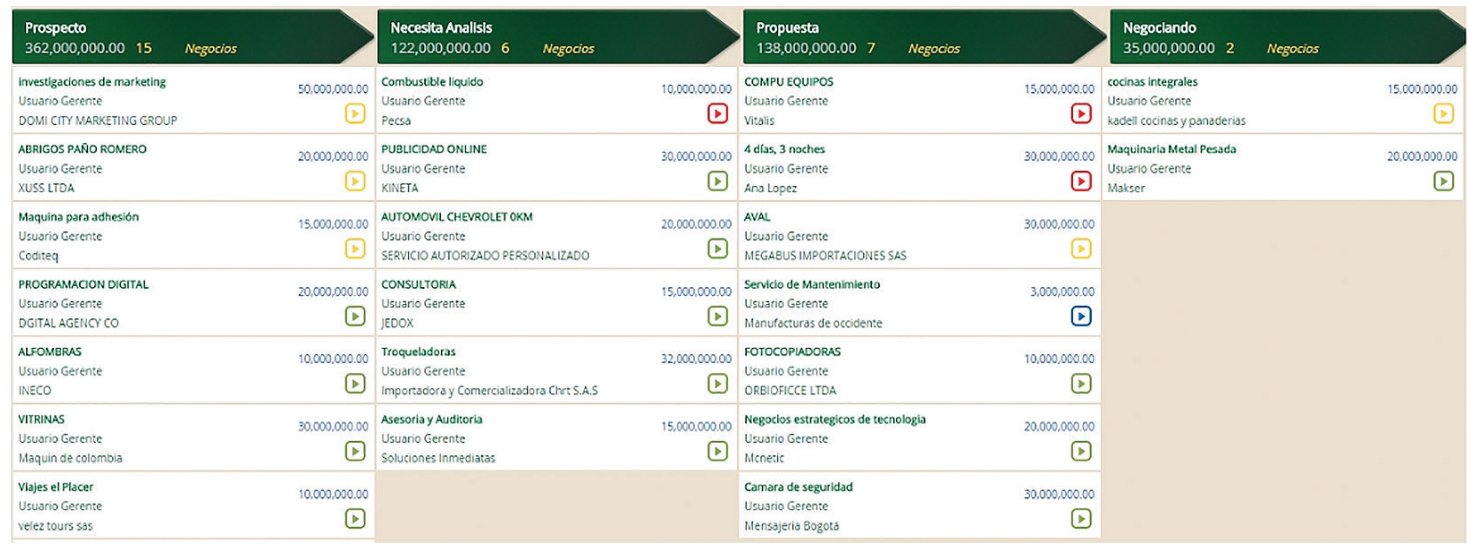

Figura 5. Aplicación del tablero Kanban a la plataforma Data CRM Fuente: [50]

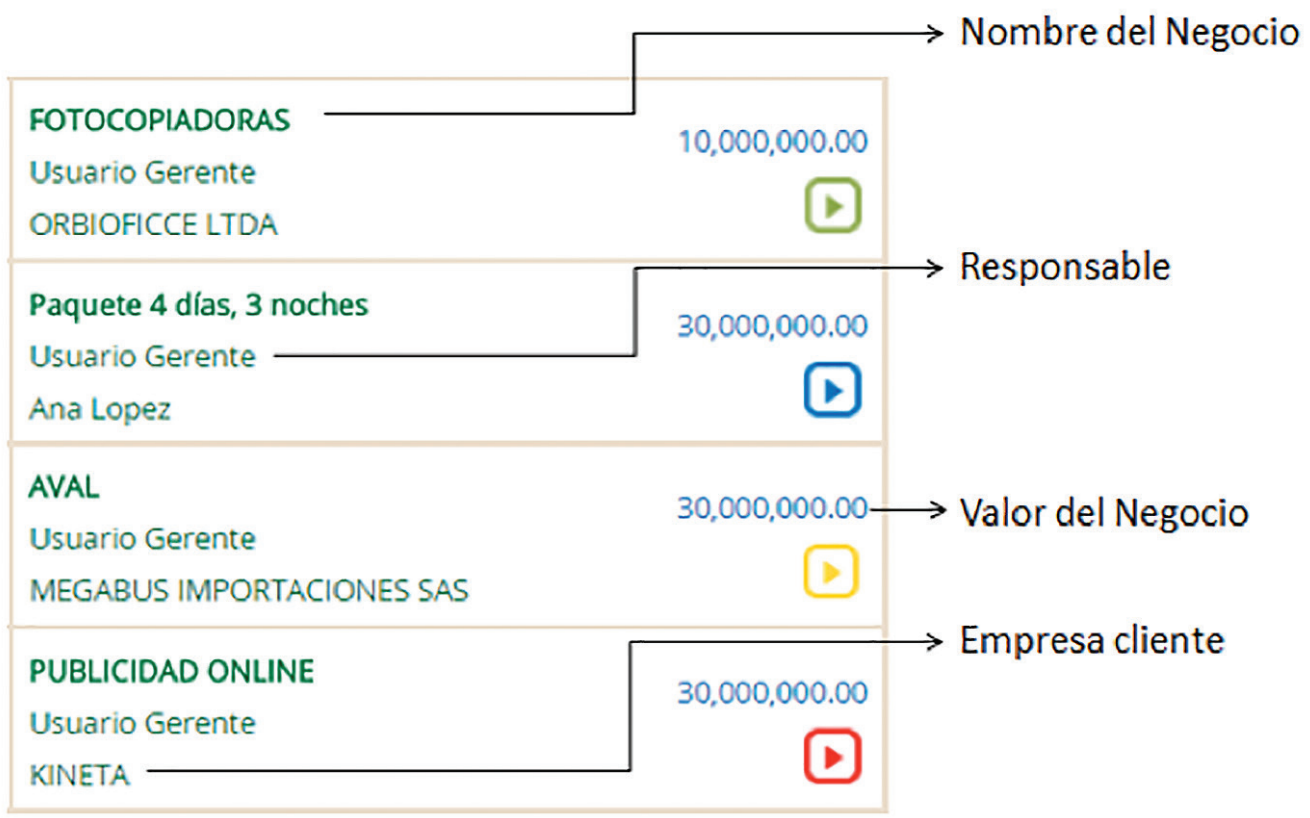

Figura 6. Descripción de los parámetros de cada tarjeta

Fuente: [50] 


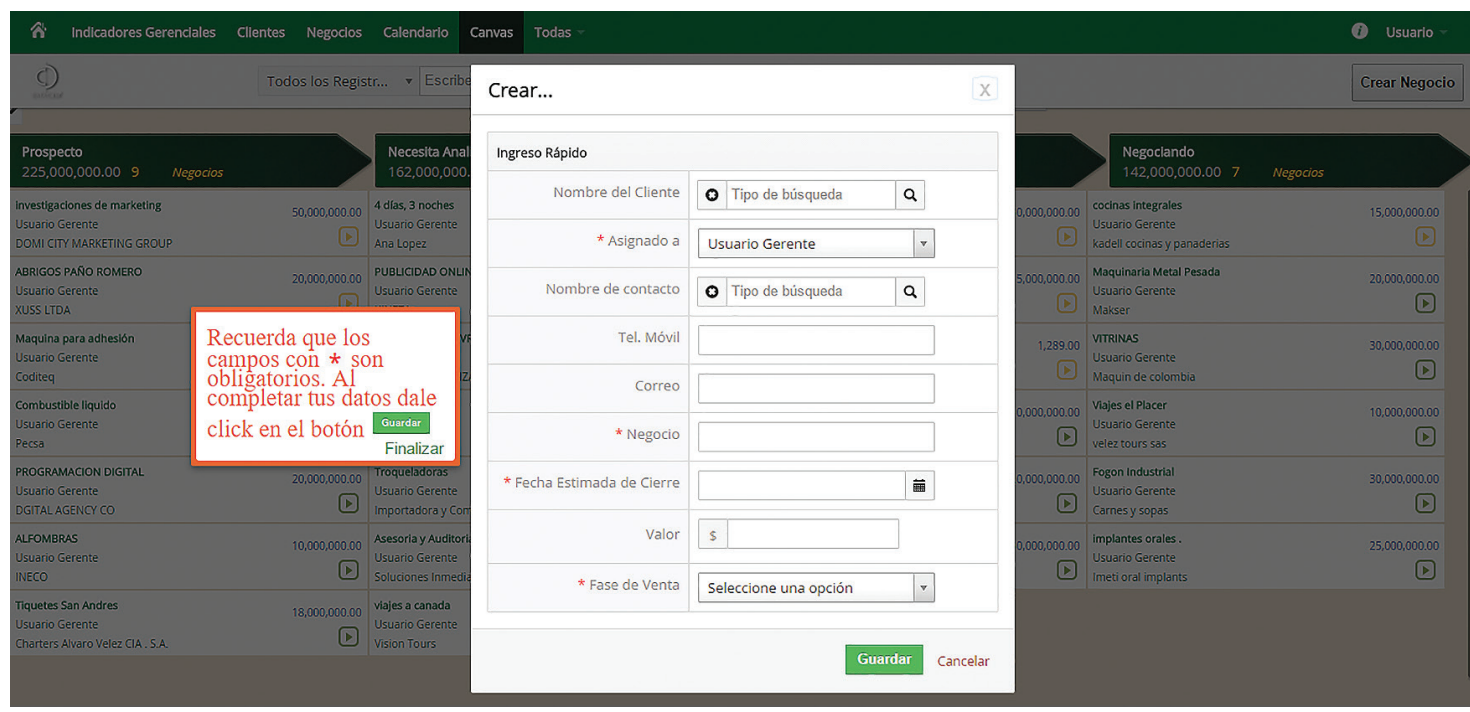

Figura 7. Formulario para crear cliente, negocio y contacto Fuente: [50]

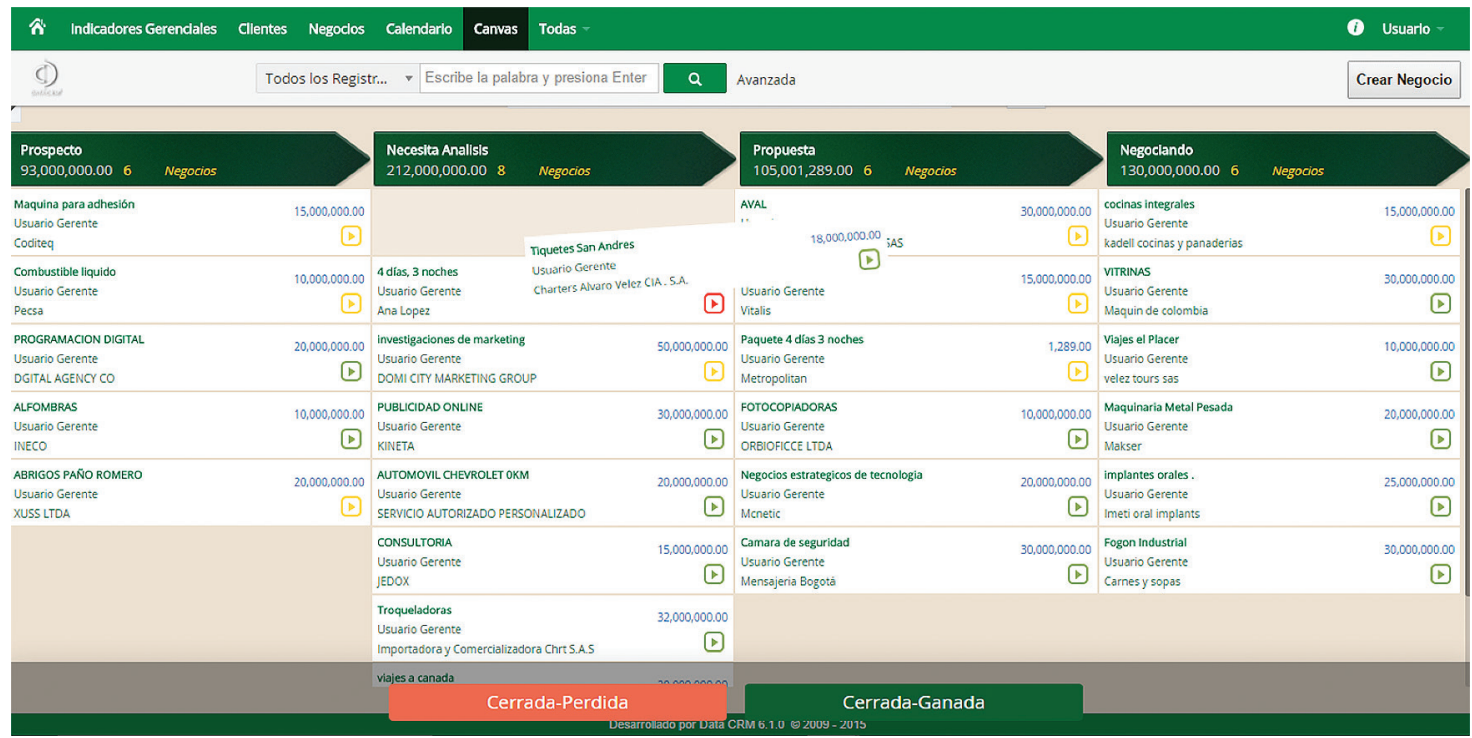

Figura 8. Tablero Canvas

Fuente: [50]

Para crear una actividad, el usuario debe dar clic en "indicador de colores" y diligenciar un formulario que, al seleccionar cualquier otro parámetro, permite actualizar o ver detalles de las actividades. Una de las características o mejoras que más ha gustado a los usuarios es la posibilidad de cambiar la fase de venta arrastrando el negocio, como se indica en la figura 8.

\section{Resultados}

Los datos obtenidos corresponden al periodo de enero-octubre del 2015 de 20 empresas usuarias de la plataforma Data CRM. La implementación de Kanban se realizó en junio del 2015, y así se distribuyó el análisis en dos momentos: enero-mayo y junio-octubre. Los datos recolectados fueron el 
número de usuarios de cada empresa y registro de actividades mensuales en la aplicación; las empresas fueron seleccionadas aleatoriamente.

Para el siguiente análisis se enumeraron las empresas aleatoriamente, es decir que los datos no corresponden al mismo orden; dichas empresas son: Свм Ingenieria, Ptesa, Insak, Sertempo, Velezcoffee, Knauf, Alliance, Doctor Arias Laparoscopia, Cidismecolsas, Comtitronic, Avance jurídico, Stemac, Insegroupsas, Soluciones Inmediatas, Visiontour, Ekip, Parking experts, Playland, Otis y Meltec. En la tabla 1 se relaciona el número de usuarios por empresa ${ }^{3}$.

Tabla 1. Número de usuarios por empresa

\begin{tabular}{|c|c|}
\hline Empresa & N. de usuarios \\
\hline Empresa 1 & 8 \\
\hline Empresa 2 & 10 \\
\hline Empresa 3 & 12 \\
\hline Empresa 4 & 5 \\
\hline Empresa 5 & 5 \\
\hline Empresa 6 & 20 \\
\hline Empresa 7 & 11 \\
\hline Empresa 8 & 3 \\
\hline Empresa 9 & 16 \\
\hline Empresa 10 & 6 \\
\hline Empresa 11 & 10 \\
\hline Empresa 12 & 4 \\
\hline Empresa 13 & 6 \\
\hline Empresa 14 & 3 \\
\hline Empresa 15 & 26 \\
\hline Empresa 16 & 8 \\
\hline Empresa 17 & 14 \\
\hline Empresa 18 & 17 \\
\hline Empresa 19 & 1 \\
\hline Empresa 20 & 47 \\
\hline
\end{tabular}

Fuente: elaboración propia

En la figura 9 se relaciona el número de actividades por empresa antes y después de la aplicación del método Kanban de la empresa 11 a la 20, $y$ de esta manera se evidencia un incremento en el uso del CRM.

3 Se aclara que este número permaneció constante en el periodo de estudio.

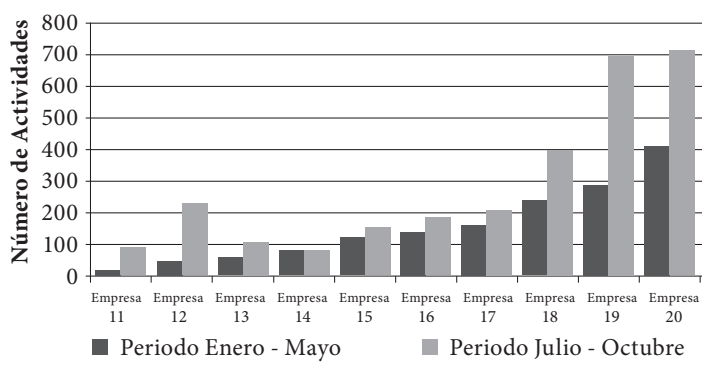

Figura 9. Actividades por empresa antes y después de la aplicación del tablero Canvas Fuente: elaboración propia

De acuerdo con la información de las 20 empresas, se puede analizar en la figura 10 que el $85 \%$ de las empresas tomadas como referencia presenta un aumento significativo del uso de la aplicación, un $10 \%$ no tiene variaciones significativas y un $5 \%$ registra un menor uso.

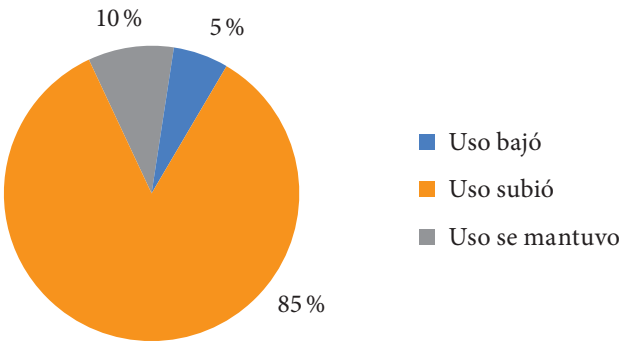

Figura 10. Variación porcentual del uso de la plataforma Fuente: elaboración propia

De las empresas que presentaron un aumento significativo de uso, el $53 \%$ evidencia un incremento entre el $20 \%$ y el $100 \%$; el $23 \%$, entre el $101 \%$ y el $180 \%$; el $18 \%$, mayor al $321 \%$; el $6 \%$, entre el $261 \%$ y el $320 \%$, y para el rango entre $181 \%$ y $260 \%$ ninguna empresa mostró este incremento, como lo indica la figura 11.

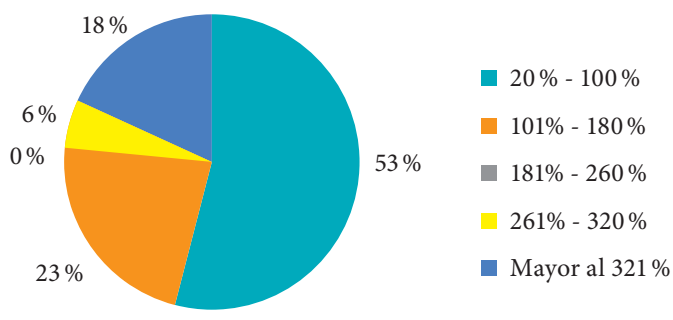

Figura 11. Rangos porcentuales del aumento de uso de la plataforma

Fuente: elaboración propia 
En la figura 12 se relaciona el número de actividades de los gerentes antes y después de la aplicación del método Kanban de la empresa 1 a la 10, lo que muestra la variabilidad del uso. Al analizar el total de las empresas, se observa que el comportamiento no representa una posición determinada respecto al incremento o decrecimiento del uso; existe un relación equivalente del $50 \%$ entre estos dos factores, por lo que se infiere que no existe un patrón de comportamiento entre el rol de los usuarios y los incrementos anteriormente relacionados.

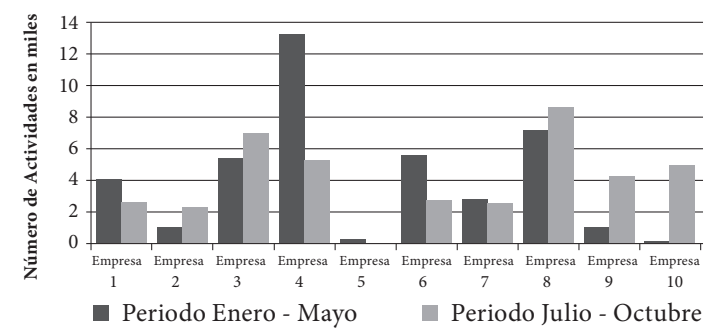

Figura 12. Actividades de los gerentes por empresa antes y después de la aplicación del tablero Canvas Fuente: elaboración propia

\section{Conclusiones}

El CRM ofertado como software de servicio es una herramienta de apoyo en los procesos de gestión de la empresa. A nivel comercial, existen diferentes aplicaciones que se acomodan a las necesidades de cada organización, desde códigos abiertos hasta aplicaciones personalizadas. Adaptar acertadamente herramientas convencionales de fácil uso e interpretación a las nuevas propuestas de software CRM tiene implicaciones positivas en el proceso de adaptación y uso eficiente.

La aplicación del tablero del método Kanban en el software CRM adiciona ventajas de control y visualización de los datos de la gestión comercial en cada fase del proceso, por lo cual se reducen tiempos de ejecución de estas operaciones, puesto que toda la información se presenta de forma ordenada y organizada, y se resaltan en primer plano datos de interés que permiten el direccionamiento a nuevas fuentes según la necesidad.

La ventaja competitiva que ofrece CRM SAAS no se limita a la adquisición de este, sino que es proporcional a la aplicabilidad dentro de la organización; por lo tanto, una interfaz amigable estimula la optimización de uso del recurso, y de esta manera beneficia a los entes que interactúan directa e indirectamente en torno a esta tendencia, como proveedores del software, usuarios del software y finalmente clientes.

Una organización al adoptar CRM no solo debe poner en funcionamiento un sistema de información, porque este solo es una herramienta tecnológica, debe también implementar una política empresarial orientada al cliente, empezando por generar cultura organizacional para involucrar esta herramienta en la cotidianidad de los usuarios, y así incluirlo en la nueva forma de gestionar sus oportunidades de negocio.

\section{Referencias}

[1] S. T. Ruehl y U. Andelfinger, "Applying Software Product Lines to create Customizable Software-as-a-Service Applications Two Kinds of Flexibility" en Conference: Software Product Lines - 15th International Conference, SPLC 2011, Munich, Alemania. Agosto 22-26, 2011, pp. 1-4. [en línea]. Disponible en: https:// www.researchgate.net/publication/220789677_Applying_software_product_lines_to_create_customizable_software-as-a-_service_applications

[2] F. Gey, D. Van Landuyt, W. Joosen y K. U. Leuven, "Continuous Evolution of Multi-tenant SAAS Applications: A Customizable Dynamic Adaptation Approach" en 2015 IEEE/ACM 7th International Workshop on Principles of Engineering Service-Oriented and Cloud Systems (PESOS), Florencia, Italia. Mayo 23, 2015, pp. 10-16. [en línea]. Disponible en: http://ieeexplore.ieee.org/xpl/login. jsp?tp=\&arnumber $=7172843 \& u r l=h t t p \% 3 \mathrm{~A} \% 2 \mathrm{~F} \%$ 2Fieeexplore.ieee.org\%2Fxpls\%2Fabs_all.jsp\%3Farnumber\%3D7172843

[3] Data CRM, "Acerca de Datacrm", 2015 [en línea]. Disponible en: https://www.datacrm.com/contenido-index-id-17-titulo-acerca-de-datacrm.htm

[4] A. S. Xanthopoulos, D. E. Koulouriotis y P. N. Botsaris, "Single-stage Kanban system with deterioration failures and condition-based preventive maintenance", Reliab. Eng. Syst. Saf., vol. 142, pp. 111122, 2015 [en línea]. Disponible en: https://www. researchgate.net/publication/277726042_Single-stage_Kanban_system_with_deterioration_failures_ and_condition-based_preventive_maintenance

[5] R. Puente y M. Cervilla, "Prácticas de la gerencia de relaciones con el cliente (CRM) en empresas venezolanas: un estudio de casos", Rev. Latinoam. Adm., vol. 39, pp. 1-28, 2007 [en línea]. Disponible en: http:// perfectaweb.com/ojs244/index.php?journal=revista\&page $=$ article\&op $=$ view\&path\%5B $\% 5 \mathrm{D}=504$ 
[6] J. Robert, V. Peñaloza, J. Alberto, S. Rueda, "Propuesta metodológica para la identificación del valor agregado como input de Lean Services en instituciones de educación superior", Rev. Ing. Ind., vol. 32, pp. 91-115, 2014 [en línea]. Disponible en: http:// fresno.ulima.edu.pe/sf/sf_bdfde.nsf/OtrosWeb/Ing32Voelkl/\$file/04-ingenieria32-VOELKL.pdf

[7] E. D. Pérez, "Sobre modas y realidades: CRM o el nuevo marketing digital", Nueva Econ. y Empres., vol. 791, pp. 55-62, abril-mayo, 2001 [en línea]. Disponible en: http://www.revistasice.com/CachePDF/ ICE_791_55-62_419D3908CAB648CD5B39E83BF1C8F59A.pdf

[8] A. Payne y P. Frow, "Customer relationship management: From strategy to implementation", Aust. New Zeal. Mark. Acad. Conf. 2005 Bus. Interact. Relationships Networks, vol. 22, no. 1, pp. 85-93, 2005 [en línea]. Disponible en: http://www.anzmac. org/conference_archive/2005/cd-site/pdfs/7-Business-Interaction-Rel/7-Payne.pdf

[9] A. M. Gil-Lafuente y C. Luis-Bassa, "La innovación centrada en el cliente utilizando el modelo de inferencias en una estrategia CRM", Investing. Eur. Dir. y Econ. la Empres., vol. 17, no. 2, pp. 15-32, 2011 [en línea]. Disponible en: http://www.sciencedirect. com/science/article/pii/S1135252312600501

[10] R. A. Soeini, B. Jafari, R. Keshavarz, A. Ganjdanesh y M. Abdollahzadeh, "Decision on Weak and Strength Points of CRM", presentado en 7th Int. Conf. Advanced Information Management and Service (ICIPM), pp. 112-120, 2011 [en línea]. Disponible en: http://ieeexplore.ieee.org./document/6222132/?reload=true

[11] S. Kabiraj, "Electronic customer relationship management: origin and opportunities", en Engineering Management Conference, 2003. IEMC '03. Managing Technologically Driven Organizations: The Human Side of Innovation and Change, Albany, New York: IEEE, 2003, pp. 484-488 [en línea]. Disponible en: http://ieeexplore.ieee.org/xpls/abs_all.jsp?arnumber $=1252320$.

[12] F. Buttle. Customer Relationship Management: Concepts and Technology. Oxford, Inglaterra: Elsevier Ltd., 2009, pp. 4-12. [en línea]. Disponible en: https:// books.google.com.co/books?hl=es\&lr=\&id=KZUttVVyEjwC\&oi=fnd\&pg=PP2\&dq=Buttle,+F.+(2009

[13] M. Valenzo, J. Arroyo y F. Avila, "Competitividad en la cadena de suministro y la gestión de relaciones con los clientes en el sector exportador aguacatero", Rev. Cimexus, vol. X, no. 1, pp. 71-88, 2015 [en línea]. Disponible en: http://cimexus.umich.mx/index.php/cim1/article/viewFile/210/171

[14] J. Sangil, “CRM ¿Filosofía o tecnología? Mitos y realidades de la orientación al cliente”, Pecvia, vol. 5, pp. 209-227, 2007 [en línea]. Disponible en: http:// revpubli.unileon.es/index.php/Pecvnia/article/ view/716

[15] M. R. L. Alonso, J.-P. Lévy-Mangin y M. A. S. Alonso, "La estrategia CRM, una visión 360 del cliente", Cienc. Ergo Sum., vol. 12, no. 1, pp. 23-34, 2005 [en línea]. Disponible en: http://www.redalyc. org/resumen.oa? id=10412103

[16] C. Araceli, "CRM Social: la orientación empresarial”, Red Marka Uima, vol. 3, pp. 3-36, 2011 [en línea]. Disponible en: http://dialnet.unirioja.es/descarga/ articulo/4125896.pdf

[17] N. Karna, I. Supriana y U. Maulidevi, "Social CRM using Web Mining”, en ICITSI 2014 (International Conference on Information Technology Systems and Innovation), Bandung - Bali, Indonesia, nov. 2014, pp. 24-27. [en línea]. Disponible en http://ieeexplore.ieee.org/xpl/articleDetails.jsp?reload=true\&arnumber $=7048275$

[18] S. Wan, C. Paris y D. Georgakopoulos, "Social Media Data Aggregation and Mining for Internet-Scale Customer Relationship Management", en 16th IEEE International Conference on Information Reuse and Intergration, Redwood City, California, 2015, pp. 39-48. [en línea]. Disponible en: http://ieeexplore.ieee.org/lpdocs/epic03/wrapper.htm?arnumber $=7300953$

[19] B. Leiner et al., "A brief history of the internet", Stud. Comput. Intell., vol. 39, no. 5, pp. 22-31, 2009 [en línea]. Disponible en: http://dl.acm.org/citation. cfm? id $=1629613$

[20] P. Mell y T. Grance, “The NIst Definition of Cloud Computing Recommendations of the National Institute of Standards and Technology", Natl. Inst. Stand. Technol. Inf. Technol. Lab., vol. 145, p. 7, 2011 [en línea]. Disponible en: http://nvlpubs.nist.gov/nistpubs/Legacy/SP/nistspecialpublication800-145.pdf

[21] I. Mangula, I. De Weerd y B. Sjaack, "Adoption of the Cloud Business Model in Indonesia: Triggers, Benefits, and Challenges", en IIWAS '12 Proceedings of the 14th International Conference on Information Integration and Web-based Applications \& Services. New York: ACM, 2012, pp. 54-63. [en línea]. Disponible en: http://doi.acm.org/10.1145/2428736.2428749

[22] B. Wu, S. Deng, Y. Li, J. Wu y J. Yin, "Reference models for SAAS oriented business workflow management systems", en International Conference on Intelligent Computing and Cognitive Informatics (ICICCI), Kuala Lumpur, 2010, pp. 242-249. [en línea]. Disponible en: http://ieeexplore.ieee.org/xpl/ articleDetails.jsp? reload=true\&arnumber $=5565952$

[23] T. Heart, "Who is out there?: exploring the effects of trust and perceived risk on SAAS adoption intentions", sIGMIS Database, vol. 41, no. 3, 
pp. 49-68, 2010. [en línea]. Disponible en: https:// dl.acm.org/purchase.cfm? id $=1851179 \&$ CFI$\mathrm{D}=865067831$ \&CFTOKEN $=44223233$

[24] H. He, "Applications deployment on the SAAS platform", en 5th International Conference on Pervasive Computing and Applications (ICPCA), Maribor, Slovenia, 2010 pp. 232-237. [en línea]. Disponible en: http://ieeexplore.ieee.org/xpl/login. jsp?tp=\&arnumber $=5704104 \& u r l=h t t p \% 3 A \% 2 F \%$ 2Fieeexplore.ieee.org\%2Fxpls\%2Fabs_all.jsp\%3Farnumber\%3D5704104

[25] J. Ju, Y. Wang, J. Fu, J. Wu y Z. Lin, "Research on Key Technology in SAAS”, en International Conference on Intelligent Computing and Cognitive Informatics (ICICCI), Kuala Lumpur, 2010, pp. 384-387. Disponible en: http://ieeexplore.ieee.org/xpl/articleDetails. jsp? reload $=$ true\&arnumber $=5565952$

[26] N. Miete, "Configurability in SAAS (software as a service) applications", Proceeding $2^{\text {nd }}$. Annu. Conf. India Softw. Eng. Conf. - ISEC '09, p. 19, 2009. [en línea]. Disponible en: http://dl.acm.org/citation.cfm? id=1506221\&CFID $=865067831 \&$ CFTOKEN $=44223233$

[27] D. Sampaio y J. Bernardino, "Open Source CRM Systems for sMes”, Int. J. Manag. Inf. Technol., vol. 3, no. 4, pp. 41-56, 2011. [en línea]. Disponible en: http:// dl.acm.org/citation.cfm?id=2641526\&dl=ACM\&coll=DL\#URLTOKEN\#

[28] G. Kulkarni, P. Khatawkar, R. Shelke, V. Solanke y R. Waghmare, “Multi-tenant SAAS cloud"' Africon, pp. 1-4, 2013 [en línea]. Disponible en: http://ieeexplore.ieee.org/document/6757684/

[29] D. Van Landuyt, S. Walraven y W. Joosen, "Variability Middleware for Multi-tenant SAAS Applications Categories and Subject Descriptors", en SPLC' 15 Proceedings of the 19th International Conference on Software Product Line. New York, USA: ACM, 2015, pp. 211-215.

[30] R. Vidhyalakshmi y V. Kumar, "Design Comparison of Traditional Application and SAAS", en International Conference on Computing for Sustainable Global Development, New Dehli, India, 2014, pp. 541-544. [en línea]. Disponible en: http://ieeexplore.ieee. org $/ x p l / \operatorname{login} . j s p ?$ tp = \&arnumber $=6828017 \& u r-$ l=http\%3A\%2F\%2Fieeexplore.ieee.org\%2Fxpls\%2Fabs_all.jsp\%3Farnumber\%3D6828017

[31] S. Xiao y G. Cheng, "Application Research of CRM Based on SAAS", en 2010 International Conference on E-Business and E-Government (ICEE), Guangzhou, China, 2010, pp. 3090-3092. [en línea]. Disponible en: http://ieeexplore.ieee.org/xpl/login. jsp?tp=\&arnumber $=5590882 \& u r l=h t t p \% 3 A \% 2 F \%-$
2Fieeexplore.ieee.org\%2Fxpls\%2Fabs_all.jsp\%3Farnumber\%3D5590882.

[32] I. Petkovic, "CRM in the cloud", en 8th International Symposium on Intelligent Systems and Informatics (SISY), Subotica, Serbia, 2010, pp. 365-370. [en línea]. Disponible en: http://ieeexplore.ieee. org/xpl/login.jsp?tp=\&arnumber=5647402\&url=http $\% 3 \mathrm{~A} \% 2 \mathrm{~F} \% 2$ Fieeexplore.ieee.org\%2Fxpls\%2Fabs_all.jsp\%3Farnumber\%3D5647402.

[33] H. S. Hu, H. Hu y H. Parsa, "Customer Relationship Management and Innovation as Performance Drivers 1 an effect on product innovation. CRM has an effect on managerial innovation. CRM has an effect on marketing innovation. CRM has", Proceedings PICMET Manag. Technol. Age, pp. 835-841, 2015. [en línea]. Disponible en: http://ieeexplore.ieee.org/document/7273244/

[34] O. Ahumada, "Dos de cada 10 empresas ya tienen 'apps' propias para sus negocios”, El Tiempo, 28 agosto, 2014 [en línea]. Disponible en http:// www.eltiempo.com/economia/empresas/empresas-con-aplicaciones-moviles/14453536

[35] Gobierno de España, Ministerio de Industria, Energía y Turismo, "Empresas que usan soluciones Software, como CRM para analizar información sobre clientes con fines de marketing," Observatorio Nacional de las telecomunicaciones y de la sI, 2015 [en línea]. Disponible en: http://www.ontsi.red.es/ ontsi/es/indicador/empresas-que-usan-soluciones-software-como-crm-para-analizar-informaci\%C3\%B3n-sobre-clientes-co.

[36] L. Columbus, "Gartner CRM Market Share Update: 47\% Of All Crm Systems Are SAAS-Based, Salesforce Accelerates Lead", Forbes, mayo 22, 2015 [en línea]. Disponible en: http://www.forbes.com/sites/louiscolumbus/2015/05/22/gartner-crm-market-share-update-47-of-all-crm-systems-are-saas-based-salesforce-accelerates-lead/\#7b00ba024e6e.

[37] L. Columbus, "Gartner сRм Market Share Update: 41\% Of CRM Systems Are SAAS-based, Salesforce Dominating Market Growth", Forbes, mayo 6, 2014 [en línea]. Disponible en: http://www.forbes.com/sites/louiscolumbus/2014/05/06/gartners-crm-market - share-update-shows-41-of-crm-systems-are-saas-based-with-salesforce-dominating-market-growth/\#3fe2a6af1d6f.

[38] Salesforce, 2015. [en línea]. Disponible en: https:// www.salesforce.com $/ \mathrm{mx} / \mathrm{crm} /$.

[39] Salesforce, "sAP", [en línea]. Disponible en: http://saperp.es/aplicaciones-sap-erp-soluciones/sap-crm/.

[40] Microsoft Dynamics CRM, "Ventajas competitivas", en Sistemas de Gestión, 2015 [en línea]. Disponible en: http://www.sistemasdegestion.com/index.php/ 
productos/microsoft-dynamics-crm/ventajas-competitivas/.

[41] Івм, "From Social Media to Social Customer Relationship Management”, 2015. [en línea]. Disponible en: http://www-935.ibm.com/services/us/gbs/thoughtleadership/ibv-social-crm-whitepaper.html

[42] Oracle, “¿Por qué Oracle CRM on Demand?”, 2015. [en línea]. Disponible en: http://www.oracle.com/ es/products/applications/crmondemand/index. html

[43] P. González y J. Molina, "Evaluación del impacto del reprocesado en los sistemas Kanban y Conwip", en XIV Congreso Ingeniería de Organización, Donostia-San Sebastián, 2010, pp. 1406-1414. [en línea]. Disponible en: http://www.adingor.es/congresos/web/articulo/detalle/a/145

[44] T. Bozheva, "Kanban: 6 prácticas para aumentar la eficiencia en proyectos TIC", Aula Dyna, vol. 88, n. ${ }^{\circ}$ 5, pp. 490-495, 2013 [en línea]. Disponible en: http://www.revistadyna.com/busqueda/kanban-6-practicas-para-aumentar-eficiencia-en-proyectos-tic

[45] N. M. Meléndez y J. A. Gallardo, “Aplicación de Sistema de Codificación IR-Kanban para Mejora en Trazabilidad de Requisitos Elicitados", Int. Work.
Adv. Softw. Eng., 2013 [en línea]. Disponible en: http://jcc2013.inf.uct.cl/wp-content/proceedings/ IWASE/Aplicacion $\% 20$ de $\% 20$ Sistema $\% 20 \mathrm{de} \% 20$ Codificacion\%20IR-Kanban\%20para\%20Mejora\%20en\%20Trazabilidad\%20de\%20Requisitos\%20 Elicitados.pdf

[46] J. Qing, P. Xue-tao y Z. Zhong, “On Solving Jit Production Problems for Small Batch Orders Based on E-Kanban Visualization", Third Int. Conf. Meas. Technol. Mechatronics Autom., pp. 757-761, 2011 [en línea]. Disponible en: http://ieeexplore.ieee.org/document/5721291/

[47] M. Bara, “3 Técnicas Esenciales para la Gestión Ágil de Proyectos”, oвs-Busins. Scho. [en línea]. Disponible en: http://www.obs-edu.com/blog-investigacion/project-management/3-tecnicas-esenciales-para-la-gestion-agil-de-proyectos/

[48] Data CRM, "Video: Datacrm - Fuerza de Venta", 2013 [en línea]. Disponible en: https://www.datacrm. com/funciones/

[49] Vtiger, "Vtiger CRM", 2015 [en línea]. Disponible en: https://www.datacrm.la/datacrm/vtigercrm/index. php

[50] “Data CRM”, 2015. [en línea]. https://www.datacrm. la/demomaker/ 